\title{
Enhancement of developmentally regulated daidzein secretion from soybean roots in field conditions as compared with hydroponic culture
}

\section{$\operatorname{AUTHOR}(\mathrm{S}):$}

Toyofuku, Miwako; Okutani, Fuki; Nakayasu, Masaru; Hamamoto, Shoichiro; Takase, Hisabumi; Yazaki, Kazufumi; Sugiyama, Akifumi

\section{CITATION:}

Toyofuku, Miwako ...[et al]. Enhancement of developmentally regulated daidzein secretion from soybean roots in field conditions as compared with hydroponic culture. Bioscience, Biotechnology, and Biochemistry 2021, 85(5): 1165-1169

\section{ISSUE DATE:}

2021-05

\section{URL:}

http://hdl.handle.net/2433/267905

\section{RIGHT:}

This is a pre-copyedited, author-produced version of an article accepted for publication in Bioscience, Biotechnology, and Biochemistry following peer review. The version of record [Miwako Toyofuku, Fuki Okutani, Masaru Nakayasu, Shoichiro Hamamoto, Hisabumi Takase, Kazufumi Yazaki, Akifumi Sugiyama, Enhancement of developmentally regulated daidzein secretion from soybean roots in field conditions as compared with hydroponic culture, Bioscience, Biotechnology, and Biochemistry, Volume 85, Issue 5, May 2021, Pages 1165-1169] is available online at:

https://doi.org/10.1093/bbb/zbab017.; The full-text file will be made open to the public on 29 January 2022 in accordance with publisher's 'Terms and Conditions for Self-Archiving'.; This is not the published version. Please cite only the published version. この論文は出版社版でありません。引用の際には出版社版をご確認ご利用ください。 
2 Enhancement of developmentally regulated daidzein secretion from soybean roots in field conditions as compared with hydroponic culture

$5 \quad$ Miwako Toyofuku ${ }^{1}$, Fuki Okutani ${ }^{1}$, Masaru Nakayasu ${ }^{1}$, Shoichiro

6 Hamamoto $^{2}$, Hisabumi Takase ${ }^{3}$, Kazufumi Yazaki $^{1}$, Akifumi Sugiyama $^{1 *}$

$9{ }^{1}$ Research Institute for Sustainable Humanosphere, Kyoto University, Gokasho, Uji,

10 611-0011, Japan, ${ }^{2}$ Graduate School of Agricultural and Life Sciences, The University

11 of Tokyo, 1-1-1 Yayoi, Bunkyo-ku, Tokyo, 113-8567, Japan, ${ }^{3}$ Faculty of

12 Bioenvironmental Science, Kyoto University of Advanced Science, Kameoka, Kyoto

13 621-8555, Japan

14

15 *Corresponding authors: Akifumi Sugiyama, tel: +81-774-38-3617, fax: +81-774-38-

16 3623, e-mail: akifumi_sugiyama@ rish.kyoto-u.ac.jp; Laboratory of Plant Gene 
17 Expression, Research Institute for Sustainable Humanosphere, Kyoto University, Uji

18 611-0011, Japan

19 
21 Enhancement of developmentally regulated daidzein secretion from

\section{soybean roots in field conditions as compared with hydroponic culture}

Analyses of metabolite secretions by field-grown plants remain scarce. We

analyzed daidzein secretion by field-grown soybean. Daidzein secretion was

higher during early vegetative stages than reproductive stages, a trend that was

a more accurate simulation of rhizosphere daidzein content.

Keywords: Daidzein; Rhizosphere; Simulation; Soybean

33 Plant specialized metabolites (PSMs) play important roles in the rhizosphere for

34 modulation of symbiotic interactions (e.g., repelling pests and pathogens and shaping 
35 microbiota), thereby promoting plant growth and improving crop production [1-3].

36 Flavonoids are a group of PSMs and consist of more than 8,000 compounds [4]. These

37 molecules function as regulators of auxin transport and reactive oxygen species and

38 protect against damage caused by ultraviolet (UV) light exposure. In legumes,

39 flavonoids are secreted from the roots to exert functions in rhizosphere plant-microbe

40 interactions, such as those necessary for defense and symbiosis [5-7].

41 Isoflavones are a subfamily of flavonoids and are found mainly in legumes [8].

42 In the rhizosphere, isoflavones such as daidzein and genistein of soybean (Glycine max)

43 and formononetin-7-O-(6"-O-malonylglycoside) of alfalfa (Medicago sativa) induce

$44 \operatorname{nod}$ genes for initiation of the nodulation process $[9,10]$. In particular, daidzein was

45 recently shown to be involved in the modulation of rhizosphere bacterial communities

46 in soybean, where this compound increased the relative abundance of the

47 Comamonadaceae family of bacteria [11]. 
49 interactions in the rhizosphere. Daidzein is the major isoflavone secreted into

50 hydroponic media, the concentration of which is higher during the soybean vegetative

51 stage than the reproductive stage [12]. Daidzein is relatively stable in soil, with a half-

52 life of about seven days, enabling the estimation of daidzein contents in the rhizosphere

53 based on the amount secreted in hydroponic cultures [13]. Whereas sorption filters or

54 glass beads have been used to collect and analyze various metabolites in the rhizosphere

$55[14,15]$, the direct measurement of secreted metabolites is technically challenging,

56 especially for field-grown plants [16]. In this study, we used the cellulose acetate

57 membrane method utilized in hydroponic culturing to analyze flavonoid secretion for

58 direct measurement of the amount of daidzein secreted by field-grown soybean plants

59 during the stages of growth.

60 All chemicals used in this study were obtained from either Wako Pure

61 Chemical Industries (Osaka, Japan) or Nacalai Tesque (Kyoto, Japan) unless otherwise 
62 stated.

64 Science, Kameoka, Kyoto, Japan (coordinates: $34^{\circ} 99^{\prime} 38^{\prime \prime}$ N, $135^{\circ} 55^{\prime} 14^{\prime \prime} E$ ). Soybean

seeds (“Tambaguro”) were sown on May 31, 2019. The plants were irrigated as needed,

66 and emerging weeds were manually removed weekly. No apparent symptoms of

67 pathogen infection were observed, and pesticides were not used. Root samples and root

68 exudates were collected on June 14 (V1 stage), July 3 (V5 stage), July 22 (V9 stage),

69 August 14 (R2 stage), September 4 (R4 stage), and October 2 (R6 stage) of 2019 [17].

70 The soil around the lateral roots was partially removed with a shovel. The lateral roots

71 were rinsed with tap water and pinched between a cellulose acetate filter (Advantec,

72 Tokyo, Japan) using a hairpin (Fig. 1A) and then covered with soil. Additionally,

73 cellulose acetate filters were placed in the bulk soil as a control. The cellulose acetate

74 filters were held in the soil for $2 \mathrm{~h}$, and then the filters and root tissues were collected.

75 All samples were transferred to the laboratory in a cool container $\left(0-10^{\circ} \mathrm{C}\right)$ within $2 \mathrm{~h}$ of 
76 collection. The root samples were stored in pure water prior to fresh weight

77 measurement. The roots and fully expanded leaves were taken from 2-week-old soybean

78 seedlings (VE stage) for the quantification of isoflavones as described previously [13].

79 Rhizosphere soil was obtained from seven plants using sterile brushes and combined

80 into one sample as described previously [18]. The samples were immediately frozen in

81 dry ice and transferred to the laboratory for storage at $-80^{\circ} \mathrm{C}$. The bulk soil was

82 sampled at least $20 \mathrm{~cm}$ away from the plant.

83 The extraction of daidzein was performed as previously described $[13,19]$. The

84 cellulose acetate filters were rinsed with tap water, and the compounds were extracted

85 twice using $1 \mathrm{ml}$ methanol with shaking on a Labo shaker BC-730 (Bio craft, Tokyo,

86 Japan) for 5 min each time. The combined supernatant from each sample was dried

87 under a nitrogen stream at $50^{\circ} \mathrm{C}$, dissolved in $150 \mu \mathrm{l}$ of methanol, and filtered through a

88 Minisart RC4 syringe filter (Sartorius, Gottingen, Germany) for LC-MS (Liquid

89 chromatography-mass spectrometry) analysis. The exudates were analyzed by UPLC- 
90 MS on an ACQUITY UPLC system (Waters Corporation) coupled with Xevo TQD.

91 The LC was performed by injecting a $2 \mu \mathrm{l}$ sample onto an ACQUITY UPLC BEH C18

92 column $(2.1 \mathrm{~mm} \times 50 \mathrm{~mm}, 1.7 \mu \mathrm{m}$; Waters Corporation $)$ at $40^{\circ} \mathrm{C}$. The LC mobile phase

93 consisted of (A) water containing $0.1 \%(\mathrm{v} / \mathrm{v})$ formic acid and (B) acetonitrile. The

94 gradient program was linear over the range of $10 \%-35 \% \mathrm{~B}, 0-1 \mathrm{~min}$; linear $35 \%-85 \%$

$95 \mathrm{~B}, 1-11 \mathrm{~min}$; isocratic $85 \% \mathrm{~B}, 11-11.1 \mathrm{~min}$; isocratic at $100 \% \mathrm{~B}, 11.1-15.5 \mathrm{~min}$; and

96 isocratic at $10 \% \mathrm{~B}, 15.5-20 \mathrm{~min}$. The flow rate was $0.2 \mathrm{ml} \mathrm{min}^{-1}$. Isoflavones were

97 detected at $260 \mathrm{~nm}$. The contents of daidzein were estimated from the peak areas in

98 comparison with calibration curves constructed using known concentrations of the

99 authentic compound.

101 tissues were pulverized in liquid nitrogen using a mortar and pestle and then freeze- 
104 through a Minisart RC4 syringe filter (Sartorius). Soil samples (1 g) were extracted in

$105500 \mu \mathrm{l}$ of methanol at $50^{\circ} \mathrm{C}$ three times $(10 \mathrm{~min}$ each) and centrifuged at $4,800 \mathrm{rpm}$ for 5

106 min. The combined supernatant from each sample was dried under a nitrogen stream at

$10750^{\circ} \mathrm{C}$ and redissolved in $150 \mu 1$ methanol. Isoflavones were analyzed by LC-MS/MS as

108 described [20].

109 The movement of daidzein secreted by a single cylindrical root was simulated

110 using a two-dimensional asymmetric system. The equations, model domains, and

111 relevant initial/boundary conditions were previously described [11]. The daidzein

112 secretion rate at the root surface was assumed to be constant $\left(1.06 \mathrm{nmol} \mathrm{m}^{2} \mathrm{~s}^{-1}\right)$, based

113 on the daidzein extraction for the roots sampled on June 14 (V1 stage). The simulation

114 period was set at 14 days with a 0.1-day time interval. The parameters used in this study

115 were summarized in Table $S 1$. A cylinder of soil with a diameter of $20 \mathrm{~cm}$ and a depth

116 of $20 \mathrm{~cm}$ with a single root of diameter $2 \mathrm{~mm}$ and length $10 \mathrm{~cm}$ in the center was set as

117 a model domain for the simulation. Root length and diameter were assumed to be 
118 constant for 14 days. The isoflavones secreted from field-grown soybean were analyzed at three

120 vegetative growth stages V2, V5, and V8, corresponding to 2, 5, and 8 weeks after

121 sowing, respectively. Moreover, samples from three reproductive growth stages R2, R4,

122 and R6, respectively corresponding to 12,15 , and 19 weeks after sowing, were

123 analyzed. Of all the detected isoflavones collected using cellulose acetate membranes

124 that adsorb flavonoid aglycones [13,19], only daidzein was identified at each growth

125 stage (Fig. 1B). The amount of secreted daidzein changed over the growth stages and

126 peaked at V5, whereas it was constant over the reproductive stages. The trend of

127 daidzein secretion was similar to that of hydroponically grown soybean. In contrast,

128 field-grown soybean secreted up to a 10,000-fold higher amount of daidzein than

129 hydroponically grown soybean (about $36 \mathrm{fmol} \mathrm{mg} \mathrm{FW}^{-1}$ day $^{-1}$ at V3) [12]. While the

130 secretion from whole roots was analyzed in the hydroponic culture media, the secretion

131 from the field-grown soybean was analyzed using a $3 \mathrm{~cm}$ root-tip section. The 
132 possibility that partial soil removal induced the isoflavone biosynthesis and increased

133 the isoflavone levels within $2 \mathrm{~h}$ is probably small because it is suggested to take more

134 than $3 \mathrm{~h}$ for the roots to accumulate isoflavones after gene induction in Arabidopsis

135 thaliana and soybean $[20,21]$. The difference in the magnitude of secretion is,

136 therefore, presumably attributable to environmental conditions, i.e., sterile hydroponics

137 vs. non-sterile field environments. The contents of isoflavones in the root tissue at

138 steady-state under the field-grown conditions were similar to those under hydroponic

139 conditions $[12,13]$, suggesting that both the isoflavone synthesis and secretions are

140 remarkably enhanced in the rhizosphere, probably due to the presence of various

141 microorganisms.

143 importance for deciphering their functions in inter-organismal interactions such as

144 chemotaxis response and nod gene induction, which are concentration-dependent $[9,22$,

145 23]; however, the distribution of PSMs remains largely unknown [24]. In our previous 
146 study, we simulated the spatiotemporal distribution of daidzein in field soil based on the

147 advection-diffusion equation [11], and we showed that daidzein distribution was limited

148 to within a few millimeters from the root surface [11]. To further refine the simulation

149 of daidzein distribution in the field, we applied the secreted rate of daidzein under field

150 conditions. The distribution of daidzein was also limited to within a few millimeters

151 from the root surface, similar to findings from the previous simulation [11]. Limited

152 daidzein distribution withn a few millimeters is likely due to the adsorption of daidzein

153 by the soil. In this simulation, the average daidzein content within 1 or $3 \mathrm{~mm}$ soils from

154 root surface was around 0.8 and $0.5 \mathrm{nmol} \mathrm{g} \mathrm{soil}{ }^{-1}$, respectively (Fig. 2A). This

155 concentration was within the range to induce nod genes in Bradyrhizobium japonicum,

156 which is reported to be more than $0.1 \mu \mathrm{M}[9,25]$. The isoflavone contents in the

157 rhizosphere and plant tissues were measured in 2-week-old soybean seedlings to

158 validate the results of this simulation. Malonylgenistin was the most predominant

159 isoflavone in the leaves at this stage, while malonyldaidzin and daidzein were 
160 accumulated in the roots (Fig. 2B). Rhizosphere soil was sampled from less than $3 \mathrm{~mm}$

161 layer from the root surface. In the rhizosphere soil, daidzein was the most abundant

162 isoflavone, and the content was about $5 \mathrm{nmol} \mathrm{g}^{\mathrm{g}}$ soil $^{-1}$ (Fig. 2C). Collectively,

163 rhizosphere modeling based on the amount secreted by field-grown soybean led to a

164 more accurate simulation of daidzein distribution than our previous simulation, i.e.

165 daidzein distribution at physiologically relevant concentrations is limited to within a

166 few millimeters from root surface.

168 the dynamics in the rhizosphere of field-grown plants is still preliminary. The dynamics

169 between proteins, metabolites, and ions in the rhizosphere have been analyzed mostly

170 using the rhizobox [23], but they should be examined in field-grown plants as well. In

171 this study, we showed that the secretion of daidzein by field-grown soybean followed

172 the same trends in terms of developmental regulation, but the amount was much higher

173 than in hydroponic condition, leading to the accurate estimation of daidzein distribution 
174 in the rhizosphere. The rhizosphere microbiome affects the secretion of metabolites

175 from roots [26]; therefore, we presume that the rhizosphere microbiome enhanced

176 daidzein secretion in the field, in addition to the effects of other both biotic and abiotic

177 stresses under field conditions. It is of particular importance to analyze the secretion of

178 PSMs in the rhizosphere of field-grown plants under various conditions and to integrate

179 the distribution of PSMs and the structure and functions of the microbiota in future

180 studies.

181

182 Acknowledgments: We thank Ms. Keiko Kanai for her technical assistance. We also

183 thank DASH/FBAS, the Research Institute for Sustainable Humanosphere, Kyoto

184 University for supporting the institutional setting.

185

186 Author Contributions: A.S. conceived and designed the research; H.T., K.Y., and A.S.

187 supervised the experiments; M.T., F.O., and M.N. conducted plant sampling and LC-

188 MS/MS analysis; S.H. conducted the simulation; M.T. and A.S. wrote the article with 
189 contributions of all authors; A.S. agrees to serve as the author responsible for contact

190 and ensuring communication.

191

192 Disclosure Statement: No potential conflict of interest was reported by the authors.

193

194 Funding: This study was supported in part by grants from JST-CREST (JPMJCR17O2

195 to A.S.) and JSPS KAKENHI (18H02313 to S.O. and A.S.) from the Research Institute

196 for Sustainable Humanosphere (Mission 1).

197

$198 \quad$ Figure Legends

199 Fig. 1 (A) Cellulose acetate membrane used to collect root exudates. Tips of lateral

200 roots were washed with pure water and pinched in a cellulose acetate membrane, which

201 was then covered with soil. The site of analysis was marked with a piece of white paper.

202 (B) Root exudation of daidzein throughout soybean growth stages. Amount per root

203 fresh weight of daidzein in root exudates $(n \geq 9)$. Significant differences $(P<0.05$;

204 Tukey-Kramer test) are indicated with various letters. Root samples and root exudates

205 were collected at three vegetative stages (V) and three reproductive stages (R). 
207 Fig. 2 Simulation of daidzein distribution in soil and isoflavone contents in the

208 rhizosphere. (A) Simulated daidzein distribution from 0 to 14 days in soil. The rate of

209 daidzein secretion from roots was assumed to be constant at each depth, and the

210 distribution at the middle of root at a depth of $5 \mathrm{~mm}$ was displayed in radial direction. It

211 is noted that vertical distribution of daidzein was not obtained in this simulation. (B)

212 Contents of isoflavones in leaves and roots $(n=3)$. (C) Contents of isoflavones in bulk

213 and rhizosphere soils at VE stage $(\mathrm{n}=3)$.

214

215 Suppmenentary Material

216 Supplementary Table 1. Parameters used in this study

\section{References}

219 [1] Chen Q, Jiang T, Liu YX, et al. Recently duplicated sesterterpene (C25) gene 
222 [2] Huang AC, Jiang T, Liu YX, et al. A specialized metabolic network selectively modulates Arabidopsis root microbiota. Science 2019;364:eaau6389.

https://doi.org/10.1126/science.aau6389.

[3] Massalha H, Korenblum E, Tholl D, et al. Small molecules below-ground: The role of specialized metabolites in the rhizosphere. Plant J 2017;90:788-807. https://doi.org/10.1111/tpj.13543.

[4] Andersen OM, and Markham KR. Flavonoids: Chemistry, biochemistry and applications. CRC Press, Boca Raton, FL 2005.

[5] Cesco S, Neumann G, Tomasi N, et al. Release of plant-borne flavonoids into the rhizosphere and their role in plant nutrition. Plant Soil 2010;329:1-25. https://doi.org/10.1007/s11104-009-0266-9. 
236 [7] Pii Y, Mimmo T, Tomasi N, et al. Microbial interactions in the rhizosphere:

Beneficial influences of plant growth-promoting rhizobacteria on nutrient acquisition process. A review. Biol Fert Soils 2015;51:403-415. Springer Verlag. https://doi.org/10.1007/s00374-015-0996-1.

240 [8] Mazur WM, Duke JA, Wähälä K, et al. Isoflavonoids and lignans in legumes:

241 Nutritional and health aspects in humans. J Nutr Biochem 1998;9(4):193-200. http://sun.ars-grin.gov/ngrlsb/.

243 [9] Kosslak RM, Bookland R, Barkei J, et al. Induction of Bradyrhizobium japonicum common nod genes by isoflavones isolated from Glycine max. Proc Natl Acad Sci U S A 1987;84:7428-7432. https://doi.org/10.1073/pnas.84.21.7428. 
249 [11] Okutani F, Hamamoto S, Aoki Y, et al. Rhizosphere modelling reveals

250 spatiotemporal distribution of daidzein shaping soybean rhizosphere bacterial community. Plant Cell Environ 2020;43:1036-1046.

https://doi.org/10.1111/pce.13708.

[12] Sugiyama A, Yamazaki Y, Yamashita K, et al. Developmental and nutritional regulation of isoflavone secretion from soybean roots. Biosci Biotechnol Biochem 2016;80:89-94. https://doi.org/10.1080/09168451.2015.1062714.

256 [13] Sugiyama A, Yamazaki Y, Hamamoto S, et al. Synthesis and secretion of isoflavones by field-grown soybean. Plant Cell Physiol 2017;58:1594-1600. https://doi.org/10.1093/pcp/pcx084.

259 [14] Phillips RP, Erlitz Y, Bier R, et al. New approach for capturing soluble root exudates in forest soils. Funct Ecol 2008;22:990-999. 
262 [15] Neumann G, Bott S, Ohler MA, et al. Root exudation and root development of lettuce (Lactuca sativa L. cv. Tizian) as affected by different soils. Front Microbiol 2014;5:2. https://doi.org/10.3389/fmicb.2014.00002.

[16] Oburger E, Jones DL. Sampling root exudates - Mission impossible? Rhizosphere 2018;6:116-133. doi: 10.1016/j.rhisph.2018.06.004.

267 [17] Fehr WR, Caviness CE. Stages of soybean development. Lowa State University Press Ames IA 1977. http://lib.dr.iastate.edu/specialreports/87.

[18] Sugiyama A, Ueda Y, Zushi T, et al. Changes in the Bacterial community of soybean rhizospheres during growth in the field. PLoS One 2014;9:100709. doi: 10.1371/journal.pone.0100709. 
276 [20] Matsuda H, Nakayasu M, Aoki Y, et al. Diurnal metabolic regulation of isoflavones and soyasaponins in soybean roots. Plant Direct 2020;4. doi: $\underline{10.1002 / \mathrm{pld} 3.286 .}$

[21] Nakabayashi R, Mori T, Nishizawa T, et al. Temporal lag between gene expression and metabolite accumulation in flavonol biosynthesis of Arabidopsis roots. Phytochem Lett 2017;22:44-48. doi: 10.1016/j.phytol.2017.09.001. japonicum to soybean exudates. Appl Environ Microbiol 1991;57:2635-2639. doi: 10.1128/aem.57.9.2635-2639.1991.

[23] Kuzyakov Y, Razavi BS. Rhizosphere size and shape: Temporal dynamics and spatial stationarity. Soil Biol Biochem 2019;135:343-360. Elsevier Ltd. https://doi.org/10.1016/j.soilbio.2019.05.011. 
290 [25] Kape R, Parniske M, Werner D. Chemotaxis and nod gene activity of

291 Bradyrhizobium japonicum in response to hydroxycinnamic acids and

292 isoflavonoids. Appl Environ Microbiol 1991;57:316-319 doi:

10.1128/aem.57.1.316-319.1991.

294 [26] Korenblum E, Dong Y, Szymanski J, et al. Rhizosphere microbiome mediates 